Check for updates

Cite this: Mater. Adv., 2020

1, 1805

Received 14th June 2020,

Accepted 23rd July 2020

DOI: 10.1039/d0ma00416b

rsc.li/materials-advances

\title{
Keratin-cinnamon essential oil biocomposite fibrous patches for skin burn care $\dagger$
}

\author{
Despoina Kossyvaki, (D) $\ddagger^{\mathrm{a}}$ Giulia Suarato, $\ddagger^{\mathrm{ab}}$ Maria Summa, ${ }^{\mathrm{b}}$ Arianna Gennari, (D) ${ }^{\mathrm{c}}$ \\ Nora Francini, ${ }^{c}$ Iosifina Gounaki, ${ }^{d}$ Danae Venieri, ${ }^{d}$ Nicola Tirelli, (D) c \\ Rosalia Bertorelli, ${ }^{b}$ Athanassia Athanassiou*a and Evie L. Papadopoulou (D) *a
}

\begin{abstract}
Novel electrospun fibrous biocomposites have been fabricated, based on two naturally derived materials, namely wool keratin and cinnamon essential oil, and their efficacy for the treatment of skin burns caused by UVB exposure is demonstrated. The cinnamon essential oil, successfully encapsulated in uniform, bead-free fibers, enhances the mechanical compliance of the composite, retains its antioxidant properties and is released from the keratin matrix upon its dissolution in an aqueous environment. The low-cytotoxicity material reduces the risk of infection from Staphylococcus aureus, Escherichia coli and Pseudomonas aeruginosa. Additionally, upon contact with UV-inflammated skin, keratin-cinnamon essential oil fibers are able to down-regulate in vivo IL- 6 and IL-1 $\beta$ cytokine production, thus limiting the inflammatory response induced by UVB radiation exposure and confirming the potential of our designed dressing as a biomedical device for burn wound care.
\end{abstract}

\section{Introduction}

Skin burns are characterized by severe tissue damage, involving morphological alterations, erythema, apoptosis, and impairment of the physiological epidermal barrier function, ${ }^{1,2}$ which may result in malfunction of microcirculation, generation of oxidative stress, and release of proinflammatory cytokines at the injured site. ${ }^{3}$ Moreover, the burnt skin loses its protective function, becoming the perfect environment for bacterial growth. ${ }^{4}$ In fact, phenomena such as infection and chronic inflammation can undermine a successful healing of the tissue. ${ }^{5,6}$ In the past decade, the design of smart dressings able to accelerate the skin burn healing process by providing antibacterial, antioxidant or anti-inflammatory features has been intensively pursued..$^{7-11}$ Generally, an active wound dressing should be biocompatible, self-adhere to both moist and dry skin surfaces, and keep the wound bed clean. ${ }^{12}$ Ideally, it

\footnotetext{
${ }^{a}$ Smart Materials Group, Istituto Italiano di Tecnologia, via Morego 30, 16163 Genova, Italy.E-mail: paraskevi.papadopoulou@iit.it, athanassia.athanassiou@iit.it

${ }^{b}$ Translational Pharmacology, Istituto Italiano di Tecnologia, via Morego 30, 16163 Genova, Italy

${ }^{c}$ Polymers and Biomaterials, Istituto Italiano di Tecnologia, via Morego 30, 16163 Genova, Italy

${ }^{d}$ School of Environment Engineering, Technical University of Crete, Akrotiri Campus, 73100 Chania, Greece

$\dagger$ Electronic supplementary information (ESI) available. See DOI: 10.1039/ d0ma00416b

\$ These authors have contributed equally to this work.
}

should also be resorbable, in order to avoid the risk of repeated injury upon removal. ${ }^{11}$ More importantly, the dressing should be able to release drugs or bioactive compounds, to counteract the onset of bacterial infections and actively support the tissue healing process. ${ }^{13-16}$

In an attempt to respond to these challenges, scientists have been using nature as a source of inspiration. Keratin, a ubiquitous biological material, can be found in the hair, wool, quills and horns of mammals (mostly composed of $\alpha$-keratins), and the feathers, claws and beaks of birds and reptiles (mostly containing $\beta$-keratins). ${ }^{17,18}$ Its abundance in nature and its ability to enhance cell proliferation and attachment ${ }^{19-22}$ make keratin an ideal material for a variety of biomedical applications, ranging from scaffolds for cell growth ${ }^{23-25}$ to drug delivery. ${ }^{26,27}$ In the field of wound management, keratin-based products have been developed and some of them have been used in acute and chronic wound management, and skin disorders. ${ }^{28}$ Given the rather poor mechanical properties of keratin and its low molecular weight (ranging from 10 to $60 \mathrm{kDa}$ ), the protein is often combined with synthetic polymers, such as PEO, ${ }^{29,30}$ or PCL and PVA, ${ }^{25,31}$ which serve as adjuvants to enhance processability.

Natural medicinal extracts can be implemented for several pharmaceutical applications. ${ }^{16,32}$ For example, Hajiali et al. ${ }^{15}$ have successfully encapsulated lavender essential oil in sodium alginate nanofibers, which led to a remarkable reduction in pro-inflammatory cytokines in a mouse skin burn model. A plant extract that since the ancient years has been exploited for its biological activities is cinnamon essential oil, an oil derived from the leaves or the bark of different types of 
cinnamon plants. Amongst them, the essential oil extracted from the bark of the Cinnamomum verum plant exhibits a wide variety of therapeutic effects, including antimicrobial activity $^{33-35}$ and antioxidant properties ${ }^{36}$ that may actively contrast skin inflammation. ${ }^{37}$ Cinnamon essential oil, mainly composed of cinnamaldehyde and eugenol aromatic compounds, is also rich in phenolic molecules and flavonoids, ${ }^{38}$ responsible for the resulting biological features. ${ }^{37,39,40}$

Herein, we present the fabrication of electrospun fibers of wool-extracted keratin with polyvinylpyrrolidone (PVP) and different concentrations of cinnamon essential oil and their assessment as dressings for skin burns. Keratin-PVP aqueous emulsions with the cinnamon essential oil were prepared for subsequent water-based, ecofriendly electrospinning, resulting in biocompatible fibrous mats. Thanks to its high surface to volume ratio, the fibrous structure would result in an adequate drug loading and release, a crucial aspect for efficient wound healing. The homogeneous distribution of the cinnamon essential oil in the polymer conveyed strong antioxidant and antimicrobial activity to the fibrous mats. Furthermore, the fibrous mats were able to release the bioactive essential oil and promote the treatment of UVB skin burns. The obtained fibers show anti-bacterial activity against both Gram (+) and Gram (-) representative bacterial species, as well as in vivo antiinflammatory effects following UVB exposure. The fibrous biocomposites and their ability to contain radiation-induced inflammation qualify our material as a potential wound dressing for skin burns.

\section{Experimental}

\subsection{Materials}

Keratin was extracted from Argentinian sheep wool (Olimpias, Italy). Cinnamon essential oil of the Cinnamomum verum species was purchased from Maitreya-Natura (Italy). Poly( $N$-vinyl pyrrolidone) (PVP) (nominally with $\overline{M_{\mathrm{w}}}=1300000 \mathrm{Da}$; from GPC analysis in DMF $\overline{M_{\mathrm{w}}}=1200000 \mathrm{Da}$ and from AF4 analysis in water $\overline{M_{\mathrm{w}}}=650000 \mathrm{Da}$, always with dispersity index $D \gg 3$; see Section $\mathrm{S} 1$ in the ESI, $\dagger$ Table S1 and Fig. S1), phosphate buffered saline (PBS), ethanol, 2,2-diphenyl-1picrylhydrazyl (DPPH), acetone, methanol, urea, $\beta$-mercaptoethanol, sodium dodecyl sulfate (SDS) and sodium azide $\left(\mathrm{NaN}_{3}\right)$ were purchased from Sigma-Aldrich.

\subsection{Keratin extraction protocol}

Argentinian sheep wool fibers were washed in a 1:1 solution of acetone:methanol ( $6 \mathrm{~h}$ under mild agitation), thoroughly washed with MilliQ water and let dry overnight at room temperature. Extraction of keratin from wool was performed following the protocol presented by Suarato et al. ${ }^{41}$ Briefly, $2 \mathrm{~g}$ of washed wool fibers were dissolved in a $7 \mathrm{M}$ urea solution $(36 \mathrm{~mL})$ with SDS $(0.107 \mathrm{M})$, using $\beta$-mercaptoethanol $(1.2 \mathrm{M})$ to remove the intra-keratin disulfide bonds. The fibers were immersed in the reagent mixtures and heated at $60{ }^{\circ} \mathrm{C}$ for $4 \mathrm{~h}$ in an oven. Subsequently, the supernatant was collected, and the fibers were pressed onto a metallic net to allow further extraction of the protein. The extracted material was dialyzed against distilled water for $48 \mathrm{~h}$ with dialysis tubes $\left(M_{\mathrm{w}}\right.$ cut-off of $3500 \mathrm{Da}$, SpectrumLabs) and then centrifuged (three times at $9000 \mathrm{rpm}$ and $4{ }^{\circ} \mathrm{C}$ for $20 \mathrm{~min}$ ), to eliminate residues and fibrous debris. The final keratin concentration was $75-80 \mathrm{mg} \mathrm{mL}^{-1}$, assessed by air evaporation.

\subsection{Preparation of the composite fibers by electrospinning}

$\mathrm{PVP} /$ keratin aqueous solutions prepared at different weight ratios $(3: 1,2: 1$, and $1: 1, \mathrm{w} / \mathrm{w})$ were stirred overnight at room temperature and were electrospun under ambient conditions. In addition, for the PVP: keratin solution with a 1:1 weight ratio, various volumes of cinnamon essential oil were added (5\%, $10 \%$ and $15 \% \mathrm{v} / \mathrm{v}$ with respect to the volume of the $\mathrm{PVP} /$ keratin solution - designated as KC5, KC10 and KC15, respectively). The resulting systems were stirred with a vortex mixer, in order to emulsify the oil-in-water phase, prior to electrospinning. The emulsions were stable for $c a$. 1 week. Electrospinning was carried out immediately after the emulsion preparation, as follows. A syringe was filled with the final solution (KC0) or emulsion (KC5, KC10 or KC15) and connected to a syringe pump (NE-1000 New Era Pump Systems, Inc.) working at a flow rate between 350 and $850 \mu \mathrm{L} \mathrm{h}^{-1}$. The stainless-steel spinneret (18-gauge needle) was clamped to the positive electrode of a high-voltage power supply (EH40R2.5, Glassman High Voltage, Inc.) applying a voltage of $24 \mathrm{kV}$ and the ground electrode was connected to an aluminum collector placed at $25 \mathrm{~cm}$ from the spinneret tip.

\subsection{Asymmetric flow field-flow fractionation (AF4)}

The composition of electrospun KC0 fibers, containing different PVP: keratin ratios, was analysed after dissolution of the fibers in deionized water $\left(10 \mathrm{mg} \mathrm{mL} \mathrm{m}^{-1}\right)$ for $2 \mathrm{~h}$ at room temperature. The AF4 system AF2000 TM (Postnova Analytics, Landsberg, Germany) was coupled online to a UV-VIS spectrophotometer at $280 \mathrm{~nm}$ (S3210, Laserchrom, Rochester, UK), a PN3609 multi-angle light scattering (MALS) system (Postnova Analytics, Landsberg, Germany), and a PN3150 refractive index (RI, Postnova Analytics, Landsberg, Germany) detector in the given order. The AF4 channel was equipped with a $350 \mu \mathrm{m}$ spacer and a $10 \mathrm{kDa}$ MWCO membrane of regenerated cellulose as an accumulation wall. A $7 \mathrm{mM}$ SDS solution supplemented with $0.02 \%$ $(\mathrm{w} / \mathrm{v})$ sodium azide $\left(\mathrm{NaN}_{3}\right)$ was filtered through a $0.1 \mu \mathrm{m}$ filter and used as the eluent. For the calibration curve, the single compounds (PVP and keratin) were individually dissolved in water with concentrations ranging from 20 to $1 \mathrm{mg} \mathrm{mL}^{-1}$. In a typical experiment, the detector flow rate was set at $1 \mathrm{~mL} \mathrm{~min} \mathrm{~m}^{-1}$ and $20 \mu \mathrm{L}$ of sample was injected over 4 minutes, setting $0.2 \mathrm{~mL} \mathrm{~min}^{-1}$ as the injection flow rate. The initial cross flow and focusing flow were set at 4.5 and $5.3 \mathrm{~mL} \mathrm{~min}^{-1}$, respectively (focusing step). For the elution step the cross flow was maintained constant at $4.5 \mathrm{~mL} \mathrm{~min}^{-1}$ for $3 \mathrm{~min}$ and then exponentially (exponent $=0.30$ ) decreased to $0 \mathrm{~mL} \min ^{-1}$ over $20 \mathrm{~min}$. Subsequently, it was kept at $0 \mathrm{~mL} \min ^{-1}$ for 8 additional min. Lastly, a rinse step was performed for $0.5 \mathrm{~min}$. The polymer concentration 
was obtained by integrating the UV/Vis signal between 7 and 35 minutes for keratin and the refractive index signal between 25 and 30 min for PVP.

\subsection{Sample characterisation}

Morphological characterisation. The morphology of the electrospun fibers was analysed by Scanning Electron Microscopy (SEM; JEOL JSM-6490L). Confocal images were recorded using a Nikon A1 confocal microscope. The specimens were excited with the $488 \mathrm{~nm}$ laser line (argon laser) and the images were collected using a green band pass filter.

Chemical analysis. Infrared spectra were obtained with a Fourier Transform Infrared Spectrometer (Equinox 70 FT-IR, Bruker), equipped with ATR (MIRacle ATR, PIKE Technologies) using a diamond crystal. All spectra were recorded in the range of $4000-600 \mathrm{~cm}^{-1}$, with a resolution of $4 \mathrm{~cm}^{-1}$, accumulating 64 scans. Micro Raman spectra were acquired under ambient conditions using a Horiba Jobin-Yvon LabRAM HR800 $\mu$ Raman spectrometer, equipped with a microscope. A $632.8 \mathrm{~nm}$ excitation line, in backscattering geometry through a $50 \times$ objective lens, was used to excite the specimens, at low power of $0.25 \mathrm{~mW}$. The grating was of 600 lines per $\mathrm{mm}$ with spectral resolution of approximately $1 \mathrm{~cm}^{-1}$.

Mechanical tests. The mechanical properties of the fiber mats were analyzed by performing stress-strain curves using a dual column tabletop universal testing System 3365, with

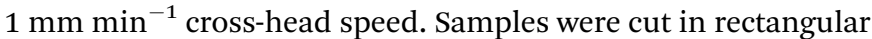
pieces with a width of $5 \mathrm{~mm}$ and an effective length of $25 \mathrm{~mm}$. From the resulting stress-strain curves the Young's modulus, tensile strength and elongation at break were calculated. An average of 7 measurements was taken for each sample.

\subsection{Drug release}

The fiber mats were studied for the release of the essential oil. Samples of $1 \mathrm{mg}$ were placed in quartz cuvettes with $3 \mathrm{~mL}$ of PBS : ethanol solution, at a volume ratio of $60: 40$, according to Hosseini et al., ${ }^{42}$ in which the electrospun fibers were not dissolved, and the absorption was measured using a UV-visible spectrophotometer (CARY200 Scan, Varian), over the course of $24 \mathrm{~h}$. Before each measurement, the cuvettes were gently shaken, in order to achieve a homogeneous dispersion of the released active compound in the solution. The release was studied following the increase of the absorption peak of cinnamaldehyde (CA), at $291 \mathrm{~nm}^{42}$ To ensure that infinite sink conditions are simulated and preserved during the course of the experiments we calculated the solubility of CA to be approximately $0.1 \mathrm{mg} \mathrm{mL}{ }^{-1}$, whereas the samples KC5, KC10 and $\mathrm{KC} 15$ reached a release of $0.004 \mathrm{mg} \mathrm{mL}^{-1}, 0.008 \mathrm{mg} \mathrm{mL}^{-1}$ and $0.010 \mathrm{mg} \mathrm{mL}^{-1}$, respectively. The experiment was carried out in triplicate and the data are expressed as cumulative percentage.

\subsection{Antioxidant activity}

The antioxidant activity of the fibers was investigated by the standard $\mathrm{DPPH}^{\bullet}$ free radical scavenging assay (RSA). Samples of $5 \mathrm{mg}$ of KC0, KC5, KC10 and KC15 fiber mats were separately placed in polystyrene cuvettes containing $2 \mathrm{~mL}$ of $0.2 \mathrm{mM}$ solution of $\mathrm{DPPH}^{\bullet}$ radical in ethanol. The decrease in the radical signal absorption was measured at $528 \mathrm{~nm}$ using a Cary 300 Scan UV-visible spectrophotometer, in the dark, at room temperature for $24 \mathrm{~h}$. All measurements were performed in triplicates. A sample with $2 \mathrm{~mL}$ of $\mathrm{DPPH}^{\bullet}$ solution was used as a control, to ensure the reagent stability. RSA was expressed as the inhibition percentage of the free radical of the samples and was calculated using eqn (1):

$$
\operatorname{RSA}(\%)=\left[\frac{(A)_{\text {control }}-(A)_{\text {sample }}}{(A)_{\text {control }}}\right] \times 100
$$

where $(A)_{\text {control }}$ is the absorption of the control sample and $(A)_{\text {sample }}$ is the absorption of the sample at different time points.

\subsection{Antibacterial activity}

The bacterial strains used for this assay were: (a) Escherichia coli reference strain DSM498 (Gram negative), (b) Pseudomonas aeruginosa (Gram negative), which was isolated from a surface water sample and was biochemically identified using the API ${ }^{\mathbb{R}}$ 20NE kit (Biomérieux) and (c) Staphylococcus aureus (Gram positive), which was isolated from a food sample (poultry) and was identified biochemically using the API ${ }^{\mathbb{R}}$ Staph test (Biomérieux). The specific bacterial species used were chosen based on their importance for public health and their frequent presence in skin wounds. All isolated strains were stored at $-80{ }^{\circ} \mathrm{C}$ in glucose solution. Bacterial inactivation for each species was performed in nutrient broth (LABM) with a bacterial concentration of $10^{2} \mathrm{CFU} \mathrm{mL} \mathrm{mL}^{-1}$. The experiment was performed for the fibrous samples (either loaded or unloaded) and the pure cinnamon essential oil as a control, following Zahid et al. ${ }^{43}$ Briefly, electrospun fibers of KC0 and KC10 were cut into small round pieces with a diameter of $1 \mathrm{~cm}$, weighing $2.8 \pm 0.5 \mathrm{mg}$. Liquid cultures were prepared for each bacterial strain in nutrient

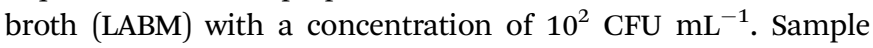
disks were added in each liquid culture and bacterial inocula were incubated at $37^{\circ} \mathrm{C}$ for $24 \mathrm{~h}$. After the incubation period, the optical density of each liquid culture was measured at $600 \mathrm{~nm}$ and viable counts were performed using nutrient agar (LABM) plates and subsequent incubation at $37{ }^{\circ} \mathrm{C}$ for $24 \mathrm{~h}$. The reduction of the bacterial population in each case was estimated based on optical density values and colony counting. Furthermore, in another set of experiments, pure cinnamon essential oil was added in the bacterial inoculum, and bacterial inactivation was recorded after a $24 \mathrm{~h}$ incubation, following the aforementioned procedure. The quantity of cinnamon oil added in each case was calculated according to the equivalent amount present in the electrospun fibers. Specifically, 1.5, 1.9 and $2.9 \mu \mathrm{L}$ of oil were added as equivalent of KC5, KC10 and KC15 samples, respectively.

\subsection{In vitro biocompatibility assay}

Primary human dermal fibroblasts (HDFa, Thermo Fisher Scientific) were cultured in T75 flasks in the presence of Medium 106 supplemented with LSGS Kit (Thermo Fisher Scientific) until 
confluency, seeded onto 24 -well plates at a density of 7000 cells per $\mathrm{cm}^{2}$, and let attach overnight in an incubator at $37{ }^{\circ} \mathrm{C}$ and with $5 \% \mathrm{CO}_{2}$. Extraction media from the various keratin-based fibrous matrices were prepared following the ISO10993-5 standard test. Briefly, fibrous samples were cut into small pieces ( $\sim 10 \mathrm{mg}$ of weight) and UV-light sterilized for 20 minutes (10 minutes per side). The fibers were immersed in $1 \mathrm{~mL}$ of cell culture medium for $24 \mathrm{~h}$ at $37{ }^{\circ} \mathrm{C}$ to obtain a stock solution which was used for the preparation of the various dilutions tested $\left(0.01,0.05,0.10\right.$, and $\left.0.50 \mathrm{mg} \mathrm{mL}^{-1}\right)$. The attached fibroblasts were then incubated with the different extraction media for $24 \mathrm{~h}$, while untreated cells were considered as control samples. MTS assay (CellTiter $96^{\circledR}$ AQueous One Solution Cell Proliferation Assay, Promega) was used to determine the cell viability following Papadopoulou et $a l^{44}$ In order to better analyze the effect of the active principle, pure cinnamon essential oil was directly applied in the culture medium, after having estimated the amount present in the corresponding loaded fibers. Prior to cell treatment, the sterile LSGS supplemented Medium 106 and essential oil emulsions were vortexed to favor oil droplet dispersion. After $24 \mathrm{~h}$, the samples were washed, and the wells were replenished with fresh culture medium, to remove the residual essential oil, and an MTS assay was performed.

Confocal imaging was carried out to observe the morphology of the cells exposed to the $\mathrm{KC}$ fiber extracts. Briefly, fibroblasts were plated onto fibronectin coated-glass coverslips at a density of 7000 cells per $\mathrm{cm}^{2}$ and treated as above-mentioned. After $24 \mathrm{~h}$, the samples were fixed with $3.7 \%$ paraformaldehyde for $20 \mathrm{~min}$, and the nuclei were stained with DAPI solution $\left(2.5 \mu \mathrm{g} \mathrm{mL}{ }^{-1}\right)$ for $15 \mathrm{~min}$ in the dark. Afterwards, the samples were permeabilized with $0.3 \%$ Triton $\mathrm{X}-100$ for $8 \mathrm{~min}$ and washed twice with PBS, prior to incubation in Alexa Fluor 564 Phalloidin (Thermo Fisher Scientific, 1:100 dilutions in PBS) for $20 \mathrm{~min}$ in the dark. Stained coverslips were then mounted with Fluoromont-G onto glass slides and imaged with a Nikon A1 confocal microscope.

\subsection{In vivo test}

Male C57BL/6J mice, 8 weeks old (Charles River, Calco, Italy), were used for the animal experiment.

Animals. The mice were grouped in ventilated cages, with free access to food and water. They were maintained under controlled conditions: temperature $\left(21 \pm 2{ }^{\circ} \mathrm{C}\right)$, humidity (50 \pm $10 \%$ ) and light (10 and $14 \mathrm{~h}$ of light and dark, respectively). All animal experiments were performed according to the guidelines established by the European Communities Council Directive (Directive 2010/63/EU of 22 September 2010) and approved by the National Council on Animal Care of the Italian Ministry of Health. All efforts were made to minimize animal suffering and to use the minimal number of animals required to obtain reliable results.

UVB-induced skin inflammation. Animals were anaesthetized with a mixture of ketamine $(10 \%)$ and xylazine $(5 \%)$ administered in a single intraperitoneal injection. The mice dorsal skin was shaved with an electric clipper, and the burn wounds were induced as previously reported. ${ }^{45}$ Briefly, mice were covered and orientated in order to have only the desired portion of skin (approximately $1.5 \mathrm{~cm}^{2}$ ) exposed to a narrow band UVB light source (TL01 fluorescent tubes, Philips, UK, $\lambda_{\text {max }}=312 \mathrm{~nm}$ ), able to produce an even field of irradiation

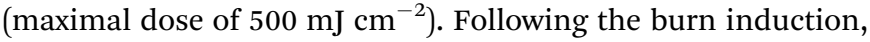
the exposed area was immediately treated by placing KC0 and KC10 fibers and protected with Tegarderm ${ }^{\mathrm{TM}}$ to avoid the removal of the matrices. The same procedure was followed for the naïve mice, which were not exposed to the UVB radiation and did not receive any treatment $(N=5$ animals for each experimental group).

Evaluation of cytokine expression. Mice were sacrificed $48 \mathrm{~h}$ after the UVB burn induction. Samples from UVB-exposed and non-exposed skin were removed and stored at $-80{ }^{\circ} \mathrm{C}$ until processing. To evaluate the expression of cytokines (IL-6 and IL-1 $\beta$ ), skin samples were homogenized and subsequently centrifuged. The obtained supernatants were collected and analyzed using an ELISA quantikine kit (R\&D system), according to the manufacturer's instructions. The cytokine concentration was normalized against the total protein content for a given sample, as measured using the bicinchoninic acid (BCA) assay (Thermo Scientific, Rockford, IL, USA). All data are presented as means \pm S.E.M. An analysis of variance was performed by ANOVA followed by a Bonferroni's multiple comparison test.

\section{Results and discussion}

\subsection{Electrospinning of PVP : keratin solutions at different mass ratios}

In order to ease the spinnability via a green, all-water electrospinning process, keratin has been combined with the biocompatible PVP, initially at a mass ratio of $3: 1$ (PVP: keratin), following a protocol previously established in our group. ${ }^{41}$ The use of the adjuvant polymer allowed the formation of stable and continuous jets under the application of a high voltage, leading to the fabrication of homogenous, bead-free fibers with average diameters of $424 \pm 137 \mathrm{~nm}$ (Fig. 1a). Increasing the concentration of the extracted keratin enabled the reduction of the synthetic and water soluble PVP in the composite solution, preserving the viscosity of the composite solution that allowed electrospinning. By increasing the keratin concentration from $40 \mathrm{mg} \mathrm{mL}{ }^{-1}$ to $80 \mathrm{mg} \mathrm{mL} \mathrm{m}^{-1}$, electrospinning with different mass ratios $(3: 1,2: 1$ and $1: 1)$ of PVP: keratin was performed. The resulting fibers presented a uniform morphology, and were rather similar in size $(466 \pm 199 \mathrm{~nm}$ and $315 \pm 89 \mathrm{~nm}$ for the $2: 1$ and $1: 1$ PVP: keratin fibers, respectively) (Fig. $1 \mathrm{~b}$ and c).

The composition of the electrospun materials was studied using a field flow fraction method; specifically, AF4 was employed with different detectors for PVP and keratin, thereby verifying the presence of the two components and simultaneously determining their actual ratio (see the ESI, $\uparrow$ Section S2, Fig. S2, S3 and Table S2; this analysis also shows no significant loss/degradation of the materials during the process). First, for each compound a calibration curve relating the sample concentration with a detector signal was obtained. PVP and keratin 

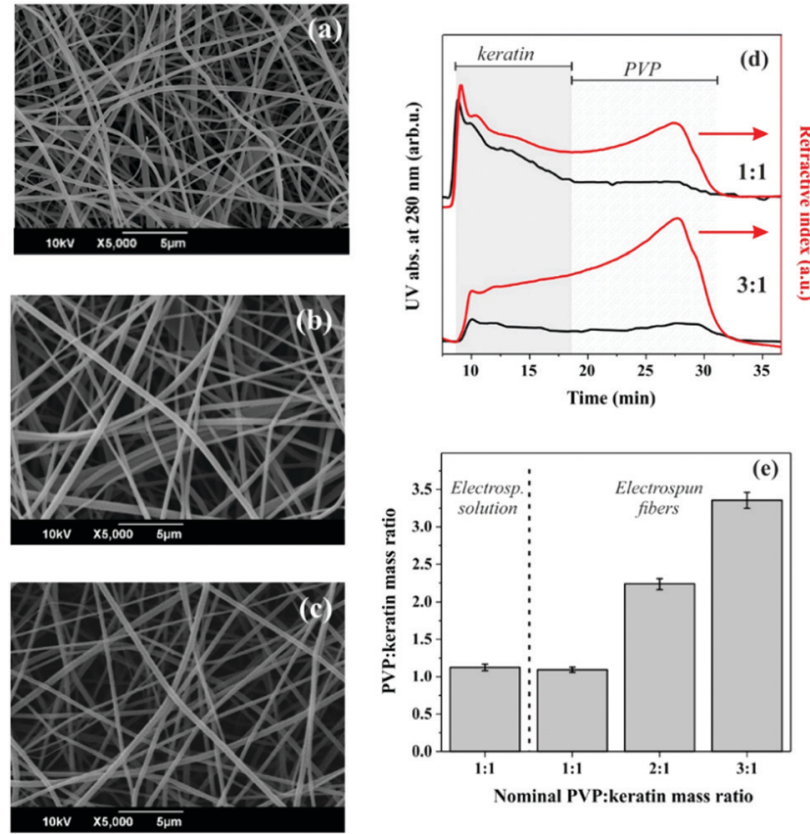

Fig. 1 SEM images of PVP: keratin fibers electrospun from solutions at different mass ratios, (a) $3: 1$, (b) $2: 1$ and (c) $1: 1$; (d) representative AF4 analyses for the 1:1 (above) and 3:1 (below) PVP:keratin fibers; the refractive index detector traces (red curves, right axis) provides peaks corresponding to the elution of both materials, but its comparison with UV absorption (black curves, left axis) allows the clearer identification of keratin. (e) The experimental values of the mass PVP/keratin mass ratio were obtained through appropriate calibration of the regions in the AF4 elugrams; the comparison between the composition of the solution used for electrospinning and the electrospun fibers (first two columns from the left) shows no significant deviation of the material composition from that of its feed. Furthermore, the mass ratio between the two components of the fibers was always close to its theoretical value.

exhibit absorption peaks at different wavelengths, allowing for the selective detection of the protein. An initial characterization conducted in parallel onto the polymer:protein $(1: 1, \mathrm{w} / \mathrm{w})$ solution prepared for the electrospinning process and the actual electrospun material revealed that the corresponding mass ratio was maintained (Fig. 1e). Subsequently, different electrospun samples, obtained from the various PVP: keratin mass ratios under study, were analysed. In Fig. 1e the PVP: keratin ratios are plotted against the nominal values. The results showed that the calculated concentration ratios finely corresponded to the theoretical ones $(1.09 \pm 0.04,2.24 \pm 0.07$, and $3.36 \pm 0.11$, for the $1: 1,2: 1,3: 1 \mathrm{PVP}$ : keratin mass ratios, respectively). Nonetheless, the presence of some keratin aggregates was detectable by the UV signal after 28 min of elution. These aggregates would also lead to a slight overestimation of the PVP concentration. Indeed, the PVP recovery appeared to be about $10 \%$ higher than the keratin one. The addition of more protein in the working solution resulted in electrospun fibers with varying mass ratios of the two components, indicating a good control of the composite formulation. Moreover, the AF4 technique, employed herein for the first time onto keratin electrospun matrices, allows a precise and straightforward characterization of the material.
More importantly, by modifying the amount of the soluble, synthetic polymer within the composite matrix, the resulting fibers were less prone to an immediate dissolution in water (data not shown). By simply tuning the fiber composition, their handling was facilitated and their application in aqueous milieu was resolved, without using any chemical or thermal crosslinking. Hence, the following characterization as well as the final in vivo assessment were carried out employing matrices obtained from PVP: keratin 1:1 mass ratios.

\subsection{Electrospinning of PVP : keratin 1:1 with cinnamon essential oil}

In order to functionalize the PVP:keratin fibers, cinnamon essential oil was introduced at different quantities $(5,10$, and $15 \% \mathrm{v} / \mathrm{v}$ with respect to the PVP:keratin solution). The small amount of SDS, residual from the protein extraction, acted as a surfactant and ameliorated the emulsification of the cinnamon essential oil in the aqueous phase, improving the stability of the emulsions, up to 1 week. All of the above led to welldefined, beadless fibers, as seen in the SEM micrographs in Fig. 2a, c and e. The average diameters were measured for KC5, KC10 and KC15, with the values of $415 \pm 98 \mathrm{~nm}, 512 \pm 194 \mathrm{~nm}$, and $531 \pm 112 \mathrm{~nm}$, respectively (Fig. 2b, d and f). This suggests a direct correlation between the amount of oil added in the emulsion systems and the size of the fabricated fibers.

Furthermore, in the confocal microscope, the autofluorescence signal of the cinnamon essential oil (in the green channel) was visibly colocalized with the bright field signal of
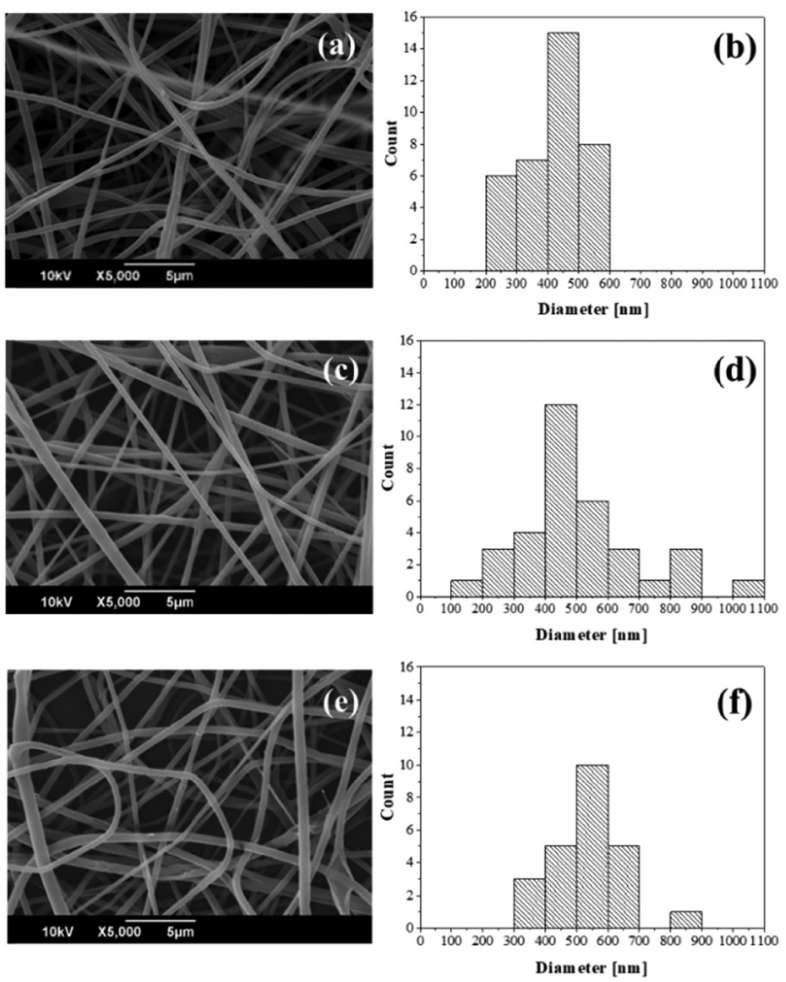

Fig. 2 SEM images and corresponding diameter distribution for ( $a$ and $b$ ) KC5, (c and d) KC10 and (e and f) KC15 electrospun fibers. 
the electrospun fibers (Fig. S4, ESI $\dagger$ ), indicating the presence of the essential oil and its homogeneous distribution within the protein-based fibers. In addition, the prepared fibrous mats presented a light-yellow color and were characterized by a rather persisting cinnamon scent, further validating the encapsulation.

\subsection{Physico-chemical analysis of the composite fibers}

The FTIR spectra of the different composite samples are shown in Fig. 3, where pristine PVP is also presented for comparison. The pristine PVP shows the main vibrational peaks at $3399 \mathrm{~cm}^{-1}$ due to the $\mathrm{O}-\mathrm{H}$ stretching, the asymmetric and


Fig. 3 (a) Comparison between the FTIR spectra of pristine Keratin, pristine PVP, and the KCO fibers; (b) FTIR spectra of the cinnamon oilloaded composite KC mats; (c) Raman spectra of the KCO mats and the composite $\mathrm{KC}$ mats. symmetric $\mathrm{CH}_{2}$ stretching modes at $2950 \mathrm{~cm}^{-1}$ and $2920 \mathrm{~cm}^{-1}$, respectively, and the amide I peak at $1643 \mathrm{~cm}^{-1}$. In addition, the bands at $1493 \mathrm{~cm}^{-1}, 1316 \mathrm{~cm}^{-1}$ and $1286 \mathrm{~cm}^{-1}$ have been assigned to the PVP backbone structure. ${ }^{13}$ Comparison of the PVP spectrum with that of KC0 reveals the presence of an overlapping of the absorption peaks between the two components. The amide I $\left(1643 \mathrm{~cm}^{-1}\right)$ band in KC0 is likely still overwhelmingly due to PVP; however, the fibers also show a clear amide II peak at $1543 \mathrm{~cm}^{-1}$, which is typical of primary amides and therefore can only be assigned to keratin. This further confirms the presence of the latter within the fibrous mats. ${ }^{46}$

In Fig. $3 \mathrm{~b}$ the vibrational peaks of KC5, KC10 and KC15 are depicted and compared to KC0. All samples exhibit vibrational peaks due to the cinnamon essential oil at $1124 \mathrm{~cm}^{-1}(\mathrm{C}-\mathrm{O}$ stretching), $973 \mathrm{~cm}^{-1}$ (C-H bending vibration), $749 \mathrm{~cm}^{-1}$ (aromatic out of plane $\mathrm{C}-\mathrm{H}$ bending) and $690 \mathrm{~cm}^{-1}$ (vibration absorption of olefins). ${ }^{47}$ In the carbonyl region, cinnamon oils present a weak peak at $1727 \mathrm{~cm}^{-1}$, a strong one at $1679 \mathrm{~cm}^{-1}$ and a weak one $1626 \mathrm{~cm}^{-1}$ and the first and the third become increasingly visible at cinnamon concentrations higher than $10 \%$ $(\mathrm{v} / \mathrm{v})$, whereas the second is overshadowed by the $\mathrm{PVP} /$ keratin amide I.

The $\mu$ Raman spectra of PVP, KC0, KC5, KC10 and KC15 fibrous mats are presented in Fig. 3c. The complementary Raman technique has been used in this study to investigate the -SS- groups of keratins. Similar to the FTIR results, comparing the spectra of KC0 and PVP the overlapping of the bands is evident. The Raman resonances associated with disulfides in pristine keratin localize at $510-520 \mathrm{~cm}^{-1}(\mathrm{~S}-\mathrm{S}$ stretching) and $650-670 \mathrm{~cm}^{-1}$ (C-S stretching); ${ }^{48}$ both of them have relatively low-intensity peaks, in particular the latter. Indeed, in most keratin-containing samples it is easy to recognize a broad peak at $500-530 \mathrm{~cm}^{-1}$ that can be assigned to keratin disulfides (either reformed during processing or -less likely- mixed with disulfides of residual mercaptoethanol). The second peak is not seen due to low intensity. ${ }^{49}$

In addition, the peaks from cinnamon essential oil are quite prominent in the spectra of KC5, KC10 and KC15. The most intense bands are present at $1003 \mathrm{~cm}^{-1}$ (2,4,6 radial in-phase stretching mode), $1599 \mathrm{~cm}^{-1}$ (ring vibration), $1631 \mathrm{~cm}^{-1}(\mathrm{C}=\mathrm{C}$ stretching), $1675 \mathrm{~cm}^{-1}$ ( $\mathrm{C}=\mathrm{O}$ stretching). For cinnamon content higher than $10 \% \mathrm{v} / \mathrm{v}$, more peaks appear at $1163 \mathrm{~cm}^{-1}, 1182 \mathrm{~cm}^{-1}$, $1254 \mathrm{~cm}^{-1}, 1580 \mathrm{~cm}^{-1}$, that are assigned to cinnamaldehyde. ${ }^{50}$

The mechanical characterization of the fibrous mats revealed that the encapsulation of the essential oils improves the ductility of the matrices. ${ }^{51,52}$ In fact, the Young's modulus decreased from $200 \pm 62 \mathrm{MPa}$ for KC0 to $48 \pm 29 \mathrm{MPa}$ for KC15, while the elongation at break remained between $2-6 \%$ for all fibrous mats, thereby showing that these systems were able to withstand handling while being conformable to a surface (i.e. a skin wound). Table S3 (ESI $\dagger$ ) reports the detailed results of the stress-strain analysis of the materials.

\subsection{Bioactive properties of the composite fiber mats}

The release of cinnamaldehyde (CA) from KC5, KC10 and KC15 fibers was investigated by UV-vis spectroscopy at different time 
points. The release profiles (Fig. 4a) showed that CA presented a burst release in the first $30 \mathrm{~min}$, followed by a very slow but sustained release profile for the next $24 \mathrm{~h}$. This behavior is of great advantage when an immediate response of the dressing is needed to deliver active principles at the wound site. ${ }^{6,9,53,54}$ After $24 \mathrm{~h}$, the KC5, KC10 and KC15 fibrous mats were able to release up to $19.6 \pm 1.9 \%, 21.1 \pm 3 \%$, and $34.6 \pm 0.5 \%$ of their total initial amount of CA, respectively (Fig. 4a). Hence, by


Fig. 4 (a) Release profile of cinnamaldehyde from KC5, KC10 and KC15 fibers up to $24 \mathrm{~h}$; (b) $\mathrm{DPPH}{ }^{\bullet}$ radical scavenging activity of KCO, KC5, KC10 and $\mathrm{KC15}$ fibers; (c) images of a cuvette before the $\mathrm{DPPH}^{\bullet}$ scavenging assay; ( $d$ and e) images of cuvettes after the DPPH ${ }^{\bullet}$ scavenging assay using fibers without and with cinnamon essential oil, respectively. The color variation is indicative of the radical inactivation. (f) Bacterial reduction of the tested bacterial strains upon incubation with the fibrous patches ( $\mathrm{KCO}$ and $\mathrm{KC10}$ ), and the pure essential oil of the corresponding concentration to KC10. tuning the amount of embedded active molecule, one can tune its release.

The $\mathrm{DPPH}^{\bullet}$ scavenging activity of the electrospun mats is reported in Fig. 4b. Surprisingly, the KC0 matrices presented an RSA of $77.3 \pm 2.5 \%$ after $24 \mathrm{~h}$. The antioxidant activity of keratin has been scarcely reported in the literature, and only specifically for keratin extracted from chicken feathers. ${ }^{18,55}$ Moreover, the PVP component does not present any radical scavenging ability. ${ }^{56}$ As expected, the presence of the cinnamon essential oil within the composite fibers enhanced their antioxidant activity by drastically decreasing the concentration of the $\mathrm{DPPH}^{\bullet}$ by $94.7 \pm 1.5 \%, 96.8 \pm 0.7 \%$ and $96.3 \pm 0.1 \%$ for the KC5, KC10 and KC15 samples, respectively, after $24 \mathrm{~h}$. Therefore, the addition of the bioactive compound (the DPPH ${ }^{\bullet}$ assay for the free cinnamon oil is reported in Fig. S5, ESI, $\dagger$ as reference) further enhanced the scavenging activity of the fibrous matrices by $19 \%$. For both $\mathrm{KC} 10$ and KC15, RSA reaches values higher than $90 \%$ within $3 \mathrm{~h}$, much sooner than for KC0 or KC5. Furthermore, complete free radical inactivation takes place with $10 \% \mathrm{v} / \mathrm{v}$ of encapsulated cinnamon essential oil, so further increase of the amount of the active molecule does not lead to higher RSA. This antioxidant boost is due to the presence of several phenolic compounds, such as cinnamaldehyde, eugenol and flavonoids. ${ }^{38,40,57,58}$

In order to assess the antibacterial features of the fabricated matrices, bacterial reduction tests were performed, with $E$. coli, $P$. aeruginosa (Gram $(-)$ ), and $S$. aureus (Gram (+)) bacterial strains, as shown in Fig. 4f. Considering first the effect of the plain PVP: keratin fibrous matrices (KC0), no reduction in the initial bacterial populations was observed for $E$. coli and $P$. aeruginosa. However, $S$. aureus exhibited a different behavior, as $24 \mathrm{~h}$-incubation with the control sample (KC0) resulted in the complete inactivation of this bacterial species (Fig. 4f, black bar). In the updated literature, studies on the antibacterial properties of keratinous materials (either extracted from wool, chicken feathers or human hair) had not led to conclusive results. For example, Caven et al. ${ }^{59}$ investigated the antibacterial nature of wool against $S$. aureus, which was observed only for the reduced components and the cuticle scales, while the cortical cells of the wool fibers appeared to be a suitable substrate for bacterial growth. In contrast, in other studies ${ }^{55,60}$ the analysis of keratin-based composite films and nanoparticles did not reveal a strong bactericidal activity. As a consequence, various interpretations of the antibacterial activity of the keratin macromolecules have been recently proposed, either based on the presence of the disulfide bonds and the content of coiled-coil random domains ${ }^{60}$ or depending on the amount of reduced protein molecules and the extraction methods employed. ${ }^{59}$ Moreover, PVP-based membranes may inhibit the biofilm formation of Staphylococcus epidermidis, affecting the transfer of nutrients and oxygen into the bacterial cells. ${ }^{61}$ In our case, the PVP-keratin fibers KC0, obtained with reduced wool keratin (mostly presenting $\alpha$-helix fractions) appeared to induce a bactericidal effect only towards $S$. aureus, in accordance with Caven et al. ${ }^{59}$

Next, the effect of the cinnamon essential oil alone on the strains was analyzed, resulting in bacterial reduction for all 
species, as expected (Fig. 4f, yellow bar). In general, the effectiveness of the essential oils may differ depending on their various chemical constituents and the structure and composition of the cell membrane pertaining to the target bacteria $($ Gram $(+)$ or Gram (-)). Thanks to their lipophilic nature, the essential oils are able to penetrate within the bacterial membrane, destabilizing the cellular architecture, and impairing cellular activities such as energy production and respiration. ${ }^{62}$ After $24 \mathrm{~h}$ of incubation with cinnamon essential oil alone, 100\% of bacterial reduction was achieved towards $S$. aureus, while the population reduction of E. coli and P. aeruginosa was 37.9 and $47.2 \%$, respectively. This difference is likely due to the hydrophobic nature of the essential oil, which can mainly and more easily penetrate the cell wall of Gram (+) bacteria (i.e. S. aureus), making the Gram (-) ones more resistant to it. ${ }^{63}$

Lastly, the effect of the cinnamon essential oil-loaded fibrous matrices (KC10, Fig. 4f, red bars) led to a population reduction of $10.1 \pm 1.5 \%$ and $26.9 \pm 7.5 \%$ for $E$. coli and $P$. aeruginosa, respectively, while the Gram $(+)$ strain $S$. aureus inactivation was reduced by $18.8 \%$. The peculiar behavior of the KC10 fibers, which show reduced $S$. aureus inactivation compared to pure essential oil and KC0 (which both show 100\% inactivation), could be explained by the efficient radical scavenging of the loaded fibers. It has been previously reported ${ }^{64-66}$ that a strong antibacterial activity can be related to the presence of free radicals, able to exert a killing oxidative stress onto the bacterial cells. ${ }^{65}$ Bacteria, like $S$. aureus, are very prone to Reactive Oxygen Species (ROS)-induced bactericidal mechanisms. ${ }^{66}$ In our case, the high antioxidant activity of KC10 (Fig. 4b, red line) results in ROS inactivation within the bacterial broth, thereby reducing the oxidative stress experienced by the bacterial cells. However, it should be pointed out that the biocidal activity of ROS is highly affected by the special features and structures of the target microorganism employed each time. For instance, Gram (+) and Gram (-) bacteria may exhibit different behavior, according to the operating conditions of the process and the materials that are used. In this sense, the thick cell wall, which is present in Gram (+) bacteria, may act as a very successful defense mechanism against the penetration of ROS, while Gram (-) species prove to be more sensitive. On the other hand, this precedence order could be reversed, due to the extra outer membrane of Gram (-) bacteria. ${ }^{67,68}$ This complex behavior is reflected in our results, as bacterial populations were reduced at various levels, whether the bacterium was Gram (+) or Gram (-) (Fig. 4f). Moreover, it is highly plausible that since ROS are no longer present in the microenvironment to carry out their bactericidal effect on the more susceptible Gram (+) $S$. aureus, the bacterial population experiences a smaller reduction (18.8\%).

Taken together, our data suggest that the effective antibacterial activity of the cinnamon essential oil-loaded PVP : keratin matrices might be the result of an interplay between the antioxidant features (via the ROS-mediated mechanism) and the presence of lipophilic chemical compounds (via the membranedestabilization mechanism). Cinnamon essential oil clearly exhibits antibacterial properties towards several bacterial species, as previously reported in the literature. ${ }^{16,69-73}$ However, direct use on burnt skin might cause sudden irritation and fast evaporation. In the herein reported strategy, the encapsulation within a fibrous matrix with high surface area would prevent the above-mentioned side effects and prolong the beneficial activity.

\subsection{In vitro biocompatibility assay}

In order to assess the cytotoxicity of the prepared matrices and their suitability for cell culture applications, primary human dermal fibroblasts (HDFa cells) were used as an in vitro model. The fibroblasts exposed for $24 \mathrm{~h}$ to different concentrations of matrix extraction media (obtained following the ISO10993-5 standard test) were completely viable, if compared to the control, untreated cells (Fig. 5a). More specifically, via MTS assay cell viabilities of $(105.8 \pm 1.6) \%,(128.1 \pm 1.0) \%,(110.4 \pm$ $0.7) \%$ and $(116.7 \pm 0.9) \%$ were registered for treatments with $0.1 \mathrm{mg} \mathrm{mL}{ }^{-1}$ of KC0, KC5, KC10 and KC15 fiber extracts, respectively. At the highest concentration tested $\left(0.5 \mathrm{mg} \mathrm{mL}^{-1}\right)$, the KC0 and KC5 matrices did not show any cytotoxic effect, while KC10 and KC15 fibers decreased the cell survival, leading to values of $(65.9 \pm 1.5) \%$ and $(76.4 \pm 1.7) \%$. The effects of direct application of free cinnamon essential oil were also considered (Fig. S6, ESI $\dagger$ ). The experiments were performed by calculating the equivalent concentrations of essential oil remaining in the various fibrous sample extracts. The results revealed a more pronounced cytotoxicity, as for higher concentrations $\left(0.5 \mathrm{mg} \mathrm{mL}^{-1}\right.$, equivalent) the cell viability dropped down to $(28.5 \pm 0.4) \%$, $(38.9 \pm$ $0.5) \%$, and $(8.15 \pm 0.2) \%$, for the corresponding KC5, KC10 and KC15 samples, respectively. Therefore, the encapsulation within the protein-based matrix mitigates the adverse biological effects in vitro of the free compounds. A confocal imaging investigation was conducted in parallel to observe the impact of the biocomposite fiber extracts $\left(0.05 \mathrm{mg} \mathrm{mL}^{-1}\right)$ onto the cellular morphology (Fig. 5b-f). For every treatment, the HDFa cells uniformly coated the glass substrates in a spread-out fashion, and presented an elongated morphology, with stretched actin fibers. No differences were noticeable between the control, untreated samples and the cells grown in the presence of either the plain or cinnamoncontaining fibers, thus confirming their suitable implementation as wound dressing materials.

\subsection{In vivo efficacy of the fibrous dressings}

Amongst the typical cutaneous manifestations after UVB-induced inflammation, there are erythema and redness, which result from vasodilation of the cutaneous blood vessels. These particular states are characterized by an upregulated production of inflammatory mediators, alteration of vascular responses, and inflammatory cell infiltration. ${ }^{74,75}$ Therefore, the use of natural products with anti-inflammatory activity, such as cinnamon essential oil, ${ }^{76,77}$ to alleviate the negative effects of UVB radiation could be of high interest.

The satisfying physico-chemical characterization presented above, the most promising antimicrobial activity, and the good biocompatibility results, led to the identification of the KC10 fibrous matrix as the most suitable for in vivo assessment. A preliminary study was conducted to determine the therapeutic 

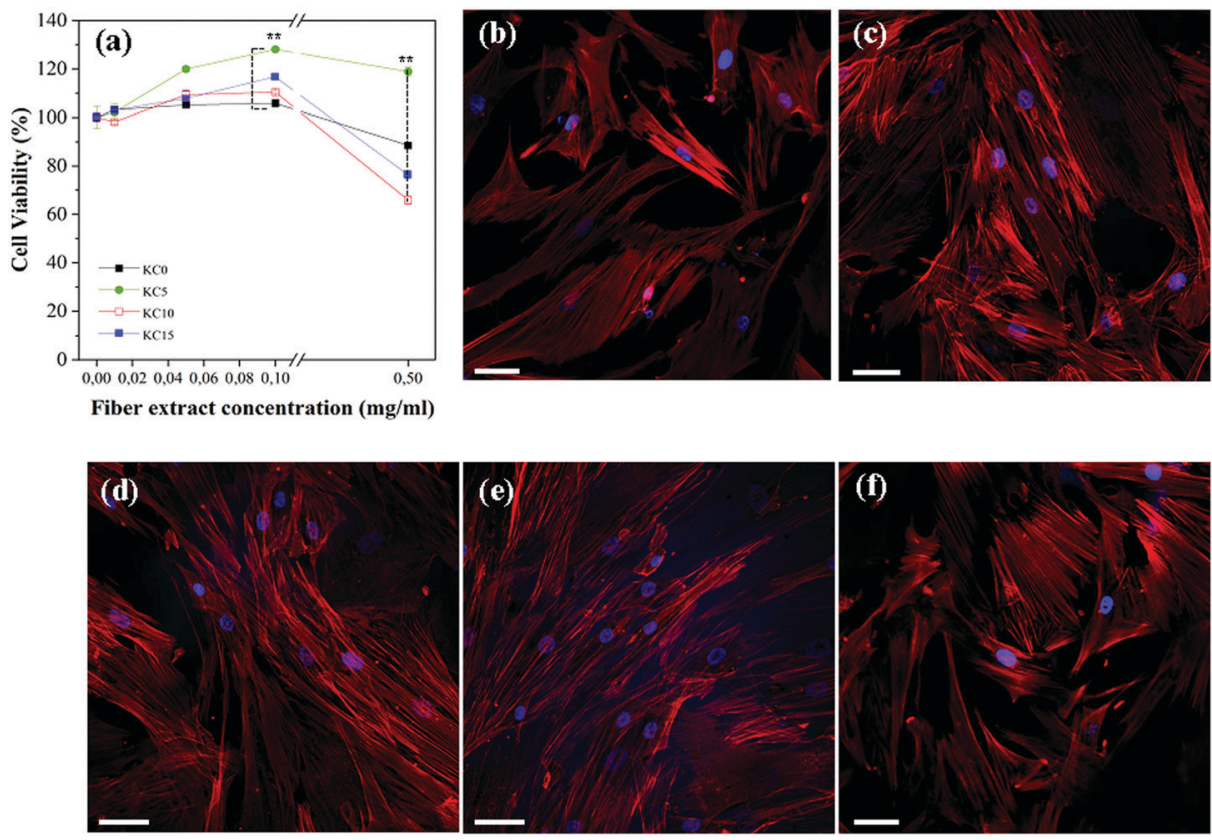

Fig. 5 Composite fiber biocompatibility study onto primary human dermal fibroblasts adult (HDFa). (a) Effect of the unloaded and loaded fiber extracts on the cell viability determined via MTS, $\left.p<0.01{ }^{(* \star}\right)$ according to a Student's $t$-test with unequal variances. Confocal imaging analysis depicting the fibroblast morphology in the (b) control samples, and in the samples treated either with $0.05 \mathrm{mg} \mathrm{mL}^{-1}$ of the (c) unloaded fiber extract, or with $0.05 \mathrm{mg} \mathrm{mL}^{-1}$ of the extract of fibers containing cinnamon essential oil fibers at a concentration of (d) $5 \%$, (e) $10 \%$, and (f) $15 \%$. Actin fibers highlighted in red (Alexa Fluor Phalloidin 546 staining) and cell nuclei in blue (DAPI staining). Scale bar $50 \mu \mathrm{m}$.

properties of the fabricated KC0 (as reference) and KC10 fibrous patches in an irradiated mouse skin model, by examining the cutaneous levels of specific inflammatory cytokines, such as IL-6 and IL-1 $\beta$, at their peak production effect after $48 \mathrm{~h}$ from the irradiation (acute phase ${ }^{78}$ ). During the in vivo experiment, the exposure of the animal skin to UVB irradiation produced evident erythema with signs of mild burn, belonging to a first degree of burn that led to visible skin injury and peak expression of cytokines within $48 \mathrm{~h}$, as previously described. ${ }^{15}$ When the burns were treated with one sole application of dressings, fibers were still present on the skin $48 \mathrm{~h}$ later, while burn marks were less visible and the erythema disappeared from the skin of the biocomposite matrices treated animals, compared to SHAM.

In Fig. 6, a significant increment of the produced cytokines can be observed for mice exposed to the UVB light without subsequent treatment (SHAM) in comparison with the ones not UVB-exposed (naïve), indicating the presence of an inflammatory state caused by the burn wound. When the biocomposite
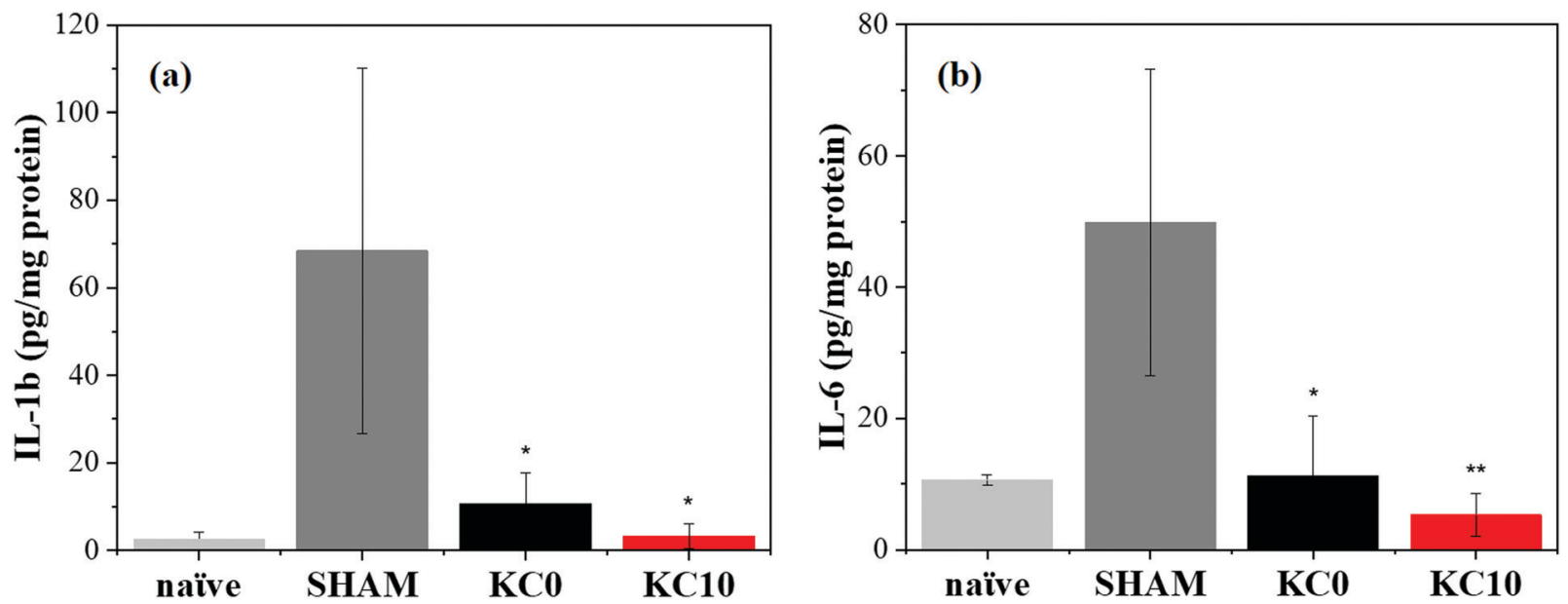

Fig. 6 In vivo wound healing results from a UV-B skin damage mouse model. Expression of the inflammatory cytokines (a) IL-1b and (b) IL- 6 for nonUVB-exposed, untreated animals (naïve), UV-B exposed, untreated animals (SHAM), UV-B exposed animals treated with KCO and UV-B exposed animals treated with KC10 $48 \mathrm{~h}$ after the irradiation. Results are presented as the average \pm S.E.M. ( $n=5$ for each group). ${ }^{\star} p<0.05$ vs. SHAM; ${ }^{* *} p<0.01$ vs. SHAM. 
patches were applied, a significant reduction in the production of inflammatory factors occurred in the mice treated with KC10 fibers compared to the untreated group. More specifically, the expression of IL-1 $\beta$ (Fig. 6a) and IL-6 (Fig. 6b) was up to 7 and 5 times lower than the UVB-irradiated group, respectively. Similar results were obtained for the group treated with KC0 fibers (6 and 4 times lower than the UVB-irradiated mice, respectively). Hence, the reduction of redness and injury correlated very well with the anti-inflammatory activities of the fibers, and the healing processes, as already demonstrated also for other biomaterials. ${ }^{15}$

The observed reduction in the expression of both the inflammatory factors considered in the study, following a single application of the composite fibers, showed that the biological reaction was contained and even prevented by our keratin-based fibers, suggesting a potential use without changing too frequently the patch. This highlights the promising and central role of the designed cinnamon-keratin-based dressings, as a platform for burn management, although further studies are needed to better understand their involvement in the management of deep burn wounds.

\section{Conclusions}

In this work, we designed and implemented a biocompatible cinnamon oil/wool keratin-based composite dressing for skin burn management. The successful dispersion of cinnamon essential oil within the polymeric matrix was confirmed with FTIR, $\mu$ Raman and confocal microscopy. When in contact with a water-like environment, the encapsulated active compounds were released from the matrices, as measured via UV-vis absorption. Our biocomposite constructs presented a notable antibacterial activity against $S$. aureus, $E$. coli and $P$. aeruginosa, and the antioxidant features of the fibers were enhanced with the addition of cinnamon essential oil. Furthermore, keratinbased patches with and without cinnamon essential oil were able to reduce in vivo the expression of pro-inflammatory factors by 5-7 fold. Therefore, this innovative natural-based material holds high potential for the healing of skin burns.

\section{Funding sources}

D. K. was supported by the Greek State Scholarships Foundation (IKY), via the Erasmus+ Program.

\section{Conflicts of interest}

There are no conflicts to declare.

\section{Acknowledgements}

Olimpias s.r.l. (Ponzano Veneto, TV, Italy) is acknowledged for kindly donating the Argentinian sheep wool. Doriana Debellis is acknowledged for the SEM images. Marco Scotto is acknowledged for his help with the confocal microscopy. D. K. acknowledges
Thi Nga Tran and Marco Contardi for the fruitful discussions and the Greek State Scholarship Foundation (IKY) for financial support.

\section{References}

1 H. Bak, S. Phil Hong, S. K. Jeong, E. H. Choi, S. E. Lee, S. H. Lee and S. K. Ahn, Int. J. Dermatol., 2011, 50, 832-837.

2 W. M. Holleran, Y. Uchida, L. Halkier-Sorensen, A. Haratake, M. Hara, J. H. Epstein and P. M. Elias, Photodermatol., Photoimmunol. Photomed., 1997, 13, 117-128.

3 B. Son, S. Lee, H. Kim, H. Kang, J. Kim, H. Youn, S. Young and B. Youn, J. Dermatol. Sci., 2019, 96, 81-89.

4 S. Kargozar, M. Mozafari, S. Hamzehlou and F. Baino, Front. Bioeng. Biotechnol., 2019, 7, 62.

5 H. Derakhshandeh, S. S. Kashaf, F. Aghabaglou, I. O. Ghanavati and A. Tamayol, Trends Biotechnol., 2018, 36, 1259-1274.

6 G. Broughton, J. E. Janis and C. E. Attinger, Plast. Reconstr. Surg., 2006, 117, 1-32.

7 K. A. Rieger, N. P. Birch and J. D. Schiffman, J. Mater. Chem. $B, 2013,1,4531-4541$.

8 M. Norouzi, S. M. Boroujeni, N. Omidvarkordshouli and M. Soleimani, Adv. Healthcare Mater., 2015, 4, 1114-1133.

9 M. B. Dreifke, A. A. Jayasuriya and A. C. Jayasuriya, Mater. Sci. Eng., C, 2015, 48, 651-662.

10 E. G. Loboa, 23 - Nanofibrous smart bandages for wound care, Elsevier Ltd, 2016, vol. 2.

11 Y. Tang, X. Lan, C. Liang, Z. Zhong, R. Xie, Y. Zhou, X. Miao, H. Wang and W. Wang, Carbohydr. Polym., 2019, 219, 113-120.

12 R. S. Ambekar and B. Kandasubramanian, Eur. Polym. J., 2019, 117, 304-336.

13 E. L. Papadopoulou, P. Valentini, F. Mussino and P. P. Pompa, Chem. Eng. J., 2018, 347, 19-26.

14 M. Contardi, D. Russo, G. Suarato, J. A. Heredia-Guerrero, L. Ceseracciu, I. Penna, N. Margaroli, M. Summa, R. Spanò, G. Tassistro, L. Vezzulli, T. Bandiera, R. Bertorelli, A. Athanassiou and I. S. Bayer, Chem. Eng. J., 2019, 358, 912-923.

15 H. Hajiali, M. Summa, D. Russo, A. Armirotti, V. Brunetti, R. Bertorelli, A. Athanassiou and E. Mele, J. Mater. Chem. B, 2016, 4, 1686-1695.

16 I. Liakos, L. Rizzello, D. J. Scurr, P. P. Pompa, I. S. Bayer and A. Athanassiou, Int. J. Pharm., 2014, 463, 137-145.

17 B. Wang, W. Yang, J. McKittrick and M. A. Meyers, Prog. Mater. Sci., 2016, 76, 229-318.

18 S. Sharma, A. Gupta, S. M. S. T. Chik, C. G. Kee, B. M. Mistry, D. H. Kim and G. Sharma, Int. J. Biol. Macromol., 2017, 104, 189-196.

19 Z. S. Thompson, N. P. Rijal, D. Jarvis, A. Edwards and N. Bhattarai, J. Visualized Exp., 2016, 108, 1-8.

20 F. Costa, R. Silva and A. R. Boccaccini, Fibrous protein-based biomaterials (silk, keratin, elastin, and resilin proteins) for tissue regeneration and repair, Elsevier Ltd, 2017.

21 A. Tachibana, Y. Furuta, H. Takeshima, T. Tanabe and K. Yamauchi, J. Biotechnol., 2002, 93, 165-170. 
22 P. Sierpinski, J. Garrett, J. Ma, P. Apel, D. Klorig, T. Smith, L. A. Koman, A. Atala and M. Van Dyke, Biomaterials, 2008, 29, 118-128.

23 J. R. Richter, R. C. De Guzman, O. K. Greengauz-Roberts and M. Van Dyke, Acta Biomater., 2012, 8, 274-281.

24 V. Verma, P. Verma, P. Ray and A. R. Ray, Biomed. Mater., 2008, 3, 025007.

25 G. Suarato, R. Bertorelli and A. Athanassiou, Front. Bioeng. Biotechnol., 2018, 6, 1-11.

26 G. Perotto, G. Sandri, C. Pignatelli, G. Milanesi and A. Athanassiou, J. Mater. Chem. B, 2019, 7, 4385-4392.

27 T. R. Ham, R. T. Lee, S. Han, S. Haque, Y. Vodovotz, J. Gu, L. R. Burnett, S. Tomblyn and J. M. Saul, Biomacromolecules, 2016, 17, 225-236.

28 R. Kelly, Keratins in wound healing, Elsevier Ltd, 2016, vol. 2.

29 A. Aluigi, C. Vineis, A. Varesano, G. Mazzuchetti, F. Ferrero and C. Tonin, Eur. Polym. J., 2008, 44, 2465-2475.

30 J. Fan, T. Lei, J. Li, P. Zhai, Y. Wang, F. Cao and Y. Liu, Mater. Des., 2016, 104, 60-67.

31 C. Wang, J. Wang, L. Zeng, Z. Qiao, X. Liu, H. Liu, J. Zhang and J. Ding, Molecules, 2019, 24, 834.

32 M. Contardi, J. A. Heredia-Guerrero, S. Guzman-Puyol, M. Summa, J. J. Benitez, L. Goldoni, G. Caputo, G. Cusimano, P. Picone, M. Di Carlo, R. Bertorelli, A. Athanassiou and I. Bayer, J. Mater. Chem. B, 2019, 7, 1384-1396.

33 D. Kalemba and A. Kunicka, Curr. Med. Chem., 2003, 10, 813-829.

34 N. Goel, H. Rohilla, G. Sing and P. Punia, J. Clin. Diagn. Res., 2016, 10, DC09-DC11.

35 A. Brochot, A. Guilbot, L. Haddioui and C. Roques, MicrobiologyOpen, 2017, 6, 1-6.

36 E. Schmidt, K. K. Co, H. A. Schloss and D. Wallerstein, J. Essent. Oil-Bear. Plants, 2006, 9, 170-182.

37 I. Husain, R. Ahmad, A. Chandra, S. T. Raza, Y. Shukla and F. Mahdi, J. Ethnopharmacol., 2018, 219, 110-116.

38 R. Ribeiro-Santos, M. Andrade, D. Madella, A. P. Martinazzo, L. de Aquino Garcia Moura, N. R. de Melo and A. Sanches-Silva, Trends Food Sci. Technol., 2017, 62, 154-169.

39 H. Chen, X. Hu, E. Chen, S. Wu, D. Julian, S. Liu, B. Li and Y. Li, Food Hydrocolloids, 2016, 61, 662-671.

40 H. Fasihi, N. Noshirvani, M. Hashemi, M. Fazilati and H. Salavati, Food Packag. Shelf Life, 2019, 19, 147-154.

41 G. Suarato, M. Contardi, G. Perotto, J. A. Heredia-Guerrero, F. Fiorentini, L. Ceseracciu, C. Pignatelli, D. Debellis, R. Bertorelli and A. Athanassiou, Mater. Sci. Eng., C, 2020, 116, 111151.

42 S. F. Hosseini, M. Zandi, M. Rezaei and F. Farahmandghavi, Carbohydr. Polym., 2013, 95, 50-56.

43 M. Zahid, E. L. Papadopoulou, G. Suarato, V. D. Binas, G. Kiriakidis, O. Moira, D. Venieri and I. S. Bayer, ACS Appl. Bio Mater., 2018, 1, 1154-1164.

44 E. L. Papadopoulou, U. C. Paul, T. N. Tran, G. Suarato, L. Ceseracciu, S. Marras, R. Arcy and A. Athanassiou, ACS Appl. Mater. Interfaces, 2019, 11, 31317-31327.
45 I. Romano, M. Summa, J. A. Heredia-Guerrero, R. Spanó, L. Ceseracciu, C. Pignatelli, R. Bertorelli, E. Mele and A. Athanassiou, Biomed. Mater., 2016, 11, 041001.

46 A. Barth, Biochim. Biophys. Acta, Bioenerg., 2007, 1767, 1073-1101. 47 Y. Q. Li, D. X. Kong and H. Wu, Ind. Crops Prod., 2013, 41, 269-278.

48 A. Kuzuhara and T. Hori, J. Mol. Struct., 2013, 1037, 85-92.

49 A. Shavandi, T. H. Silva, A. A. Bekhit and A. E. D. A. Bekhit, Biomater. Sci., 2017, 5, 1699-1735.

50 G. N. Andreev, B. Schrader, H. Schulz, R. Fuchs, S. Popov and N. Handjieva, Anal. Bioanal. Chem., 2001, 371, 1009-1017.

51 H. Atri, E. Bidram and D. E. Dunstan, Materials, 2015, 8, 7472-7485.

52 M. Yahyaoui, O. Gordobil, R. Herrera, M. Abderrabba and J. Labidi, React. Funct. Polym., 2016, 109, 1-8.

53 J. Wang, V. Planz, B. Vukosavljevic and M. Windbergs, Eur. J. Pharm. Biopharm., 2018, 129, 175-183.

54 S. Guo and L. A. DiPietro, J. Dent. Res., 2010, 89, 219-229.

55 M. Sundaram, R. Legadevi, N. Afrin Banu and V. Gayathri, Eur. J. Biotechnol. Biosci., 2015, 3, 1-5.

56 M. Ignatova, N. Manolova, I. Rashkov and N. Markova, Int. J. Pharm., 2018, 545, 342-356.

57 P. V. Rao and S. H. Gan, Evidence-Based Complementary Altern. Med., 2014, 2014, 1-2.

58 K. N. Prasad, B. Yang, X. Dong, G. Jiang, H. Zhang, H. Xie and Y. Jiang, Innov. Food Sci. Emerg. Technol., 2009, 10, 627-632.

59 B. Caven, B. Redl and T. Bechtold, Text. Res. J., 2019, 89, 510-516.

60 C. D. Tran, F. Prosencyes, M. Franko and G. Benzi, Carbohydr. Polym., 2016, 151, 1269-1276.

61 N. Bou Haidar, S. Marais, E. Dé, A. Schaumann, M. Barreau, M. G. J. Feuilloley and A. C. Duncan, Mater. Sci. Eng., C, 2020, 106, 110130.

62 M. K. Swamy, M. S. Akhtar and U. R. Sinniah, Evidence-Based Complementary Altern. Med., 2016, 2016, 3012462.

63 A. Man, L. Santacroce, R. Jacob, A. Mare and L. Man, Pathogens, 2019, 8, 1-11.

64 T. Paul, A. Mandal, S. M. Mandal, K. Ghosh, A. K. Mandal, S. K. Halder, A. Das, S. K. Maji, A. Kati, P. K. D. Mohapatra, B. R. Pati and K. C. Mondal, Appl. Biochem. Biotechnol., 2015, 175, 3371-3386.

65 M. Y. Memar, R. Ghotaslou, M. Samiei and K. Adibkia, Infect. Drug Resist., 2018, 11, 567-576.

66 D. Díaz-García, P. R. Ardiles, M. Díaz-Sánchez, I. MenaPalomo, I. del Hierro, S. Prashar, A. Rodríguez-Diéguez, P. L. Páez and S. Gómez-Ruiz, J. Inorg. Biochem., 2020, 203, 110912.

67 T. Makropoulou, P. Panagiotopoulou and D. Venieri, J. Chem. Technol. Biotechnol., 2018, 93, 2518-2526.

68 I. Levchuk, T. Homola, J. Moreno-Andres, J. J. Rueda-Marquez, P. Dzik, M. A. Morinigo, M. Sillanpaa, M. A. Manzano and R. Vahala, Sol. Energy, 2019, 191, 518-529.

69 S. Chouhan, K. Sharma and S. Guleria, Medicines, 2017, 4, 58.

70 Z. I. Yildiz, M. E. Kilic, E. Durgun and T. Uyar, J. Agric. Food Chem., 2019, 67, 11066-11076. 
71 M. Laranjo, A. M. Fernández, L. Ana, C. A. Santos, M. E. Potes and M. Elias, J. Food Process. Preserv., 2019, e14278.

72 D. Kaic and A. Rosario, Int. Food Res. J., 2019, 26, 1103-1110.

73 Y. El Atki, I. Aouam, K. Nayme and B. Lyoussi, J. Adv. Pharm. Technol. Res., 2019, 10, 63-67.

74 L. L. Hruza and A. P. Pentland, J. Invest. Dermatol., 1993, 100, S35-S41.
75 C. Kuanpradit, Y. Jaisin, S. Jungudomjaroen and S. A. Mitu, Int. J. Mol. Med., 2017, 39, 1083-1090.

76 M. R. Farahpour, A. Amniattalab and H. Hajizadeh, Afr. J. Biotechnol., 2012, 11, 15068-15071.

77 Z. Hussain, J. A. Khan, A. Arshad, P. Asif, H. Rashid and M. I. Arshad, Biomed. Pharmacother., 2019, 109, 2285-2292.

78 I. W. Nasr, C. A. Massaad, B. Sa, N. E. Saade, S. J. Jabbur and S. A. Kanaan, Br. J. Pharmacol., 2000, 131, 1317-1324. 